\title{
OPEN Development and implementation of SafeMedWaste, a chemical denaturant for non-hazardous disposal of controlled medications
}

\author{
Emma Leishman ${ }^{1 \bowtie}$, Yizhong Wang ${ }^{1}$, Reddy Channu ${ }^{1}$, Evan Boyst ${ }^{1}$, Marshall Hartmann ${ }^{2}$ \& \\ Justin Stas ${ }^{2}$ \\ Substance use disorders are a significant public health issue. Options to dispose of controlled \\ medications are limited, increasing the risk of diversion. Providing an alternative for disposal, a \\ chemical denaturant, SafeMedWaste, was designed to destroy controlled substances irreversibly \\ and safely be placed in non-hazardous landfills. Via HPLC-MS, four formulations of SafeMedWaste \\ were tested with 34 different liquid controlled medications from DEA schedules I-V. Beta testing \\ assessed the efficacy of SafeMedWaste in a clinical setting and on waste generated in a manufacturing \\ setting. Furthermore, a formulation of SafeMedWaste was tested on solid controlled medications. \\ All 34 of the liquid medications tested (e.g., amphetamine, diazepam, fentanyl, ketamine) were \\ fully destroyed in SafeMedWaste within 2-24 h. Analysis of a beta test sample of SafeMedWaste \\ containing fentanyl, midazolam, and morphine waste collected in a hospital showed full denaturation \\ of these drugs in $\mathbf{2 4} \mathrm{h}$. Variants of SafeMedWaste were optimized to denature six different controlled \\ substance waste samples from a manufacturing facility. In contrast to side-by-side studies with a \\ charcoal disposal system using the same drugs, SafeMedWaste fully inactivated and destroyed the \\ controlled substances in the waste streams. Another formulation of SafeMedWaste was tested on \\ solid medications, which were fully denatured in 48-72 h. In conclusion, SafeMedWaste irreversibly \\ denatures controlled medications that present a problem in our society.
}

Drug overdose is currently the leading cause of accidental death in the United States, surpassing deaths caused by road traffic collisions ${ }^{1}$. Millions of Americans struggle with substance use disorders, affecting entire families and communities and creating a costly public health burden ${ }^{2}$. While considerable steps have been made in our understanding of the etiology of substance abuse disorders ${ }^{3,4}$ and in reducing the prescribing of controlled substances such as opioids $s^{5,6}$, these drugs continue to have medical uses and will be part of our pharmacopeia for the foreseeable future.

In cases of prescription drug abuse, leftover and unused medication is often diverted away from its intended user. This can happen at multiple levels: in the home, in the clinic, and in the factory. In the home, unused medications are frequently left in cabinets, thrown in the trash, or flushed down the toilet ${ }^{7-10}$. It is estimated that half of people with opioid use disorder obtain drugs from friends and family ${ }^{11}$. Even when flushed, the chemical composition of the active drug does not change, adding adverse environmental impacts to the problem ${ }^{12-14}$. Among healthcare professionals, the prevalence of substance use disorder is estimated to be around $15 \%$ for pharmacists, $10 \%$ for nurses, and $8 \%$ for physicians ${ }^{15}$. While rates of substance abuse might not be significantly higher in healthcare professionals compared to the general population, there is an increased risk to the public when these professionals are impaired by drugs or alcohol ${ }^{16}$. Diversion of drugs meant for patients and of leftover medications by nurses and physicians is an unfortunate source of opioids and other drugs of abuse $\mathrm{e}^{17}$. In addition, there is an unmet need for disposal options for controlled medication manufacturing facilities. Currently, the only DEA-approved method of controlled substance disposal is burning the waste at an approved location ${ }^{18,19}$, which can be costly for the manufacturer and involves having to transport the waste to an off-site facility. The transportation and subsequent incineration of this waste stream further degrades the environment.

To meet the need for a safe and effective method of controlled medication disposal, a blend of active chemical denaturants combined with solidifying agents, known as SafeMedWaste ${ }^{20}$, was tested on over thirty liquid

${ }^{1}$ Avomeen, Ann Arbor, MI, USA. ${ }^{2}$ Okra Medical, Inc., Johns Island, SC, USA. ${ }^{\boxplus}$ email: eleishman@avomeen.com 
drugs from DEA schedules I-V. Denaturation was measured by LC-MS, showing full destruction of the active pharmaceutical ingredient (API) in approximately $24 \mathrm{~h}$. Demonstrating the real-world applications of this drug disposal system, beta tests were performed in a hospital setting and in a manufacturing setting, showing successful denaturation of drugs in the waste streams. A formulation of SafeMedWaste denatured a representative set of controlled solid medications within $72 \mathrm{~h}$, potentially expanding the scope of SafeMedWaste to a residential setting. This data demonstrates that SafeMedWaste is an effective, versatile product, with formulations to meet the needs of a wide range of controlled substance waste. Additional beta testing is planned to evaluate SafeMedWaste in the residential setting before full implementation of this formulation.

\section{Methods}

Liquid controlled medications and solutions of SafeMedWaste active ingredients. SafeMedWaste contains a mixture of active denaturants, which are oxidizing agents and solidifying agents. To determine the most effective blend of active ingredients for SafeMedWaste, four candidate formulations were tested, referred to as SafeMedWaste I-IV. There are three primary denaturants used in SafeMedWaste: potassium permanganate $\left(\mathrm{KMnO}_{4}\right)$, sodium dichloroisocyanurate (NaDCC), and trichloroisocyanuric acid (TCI). SafeMedWaste I contained all three denaturants, whereas SafeMedWaste variants II-IV used a single active denaturant. Specifically, the active components of SafeMedWaste II-IV were $\mathrm{NaDCC}$, TCI, and $\mathrm{KMnO}_{4}$, respectively. For analytical testing, SafeMedWaste I-IV active ingredients were diluted in purified water. Solutions were prepared fresh weekly.

Alprazolam, amphetamine, butorphanol, chlordiazepoxide, clonazepam, cocaine, codeine, diazepam, ephedrine, fentanyl, gabapentin, hydrocodone, hydromorphone, ketamine, lorazepam, meperidine, methadone, methamphetamine, methylphenidate, midazolam, morphine, nalbuphine, oxycodone, pentobarbital, phenobarbital, pregabalin, propofol, propoxyphene, remifentanil, sufentanil, temazepam, THC, tramadol, and zolpidem were provided as analytical reference standards at concentrations of $1 \mathrm{mg} / \mathrm{mL}$ in methanol. On the day of the experiment, $1 \mathrm{mg} / \mathrm{mL}$ drug stocks were diluted to $100 \mu \mathrm{g} / \mathrm{mL}$ in methanol. $500 \mu \mathrm{L}$ of each $100 \mu \mathrm{g} / \mathrm{mL}$ solution were placed in two separate glass vials. SafeMedWaste active ingredient solution was added to one of the two vials, whereas the same volume of water was added to the other vial to serve as a control. Contents of both vials were mixed well. After $\sim 24 \mathrm{~h}$, up to $5 \mathrm{~mL}$ methanol were added to each vial and mixed well. Samples were individually filtered into separate HPLC vials for analysis.

Denaturation of fentanyl in the full formulation of SafeMedWaste I. After establishing the levels of active ingredients required to denature each drug, the next experiment tested whether the addition of the solidifying agents to SafeMedWaste I affected the denaturation of fentanyl. $2.5 \mathrm{~mL}$ of $200 \mu \mathrm{g} / \mathrm{mL}$ fentanyl (dissolved in methanol) were transferred to two separate vials. Vial 1 was empty to serve as a control, whereas Vial 2 contained an aliquot of $165 \mathrm{mg}$ SafeMedWaste I. $2.5 \mathrm{~mL}$ water were added to each vial. Vials were capped, shaken, and left alone for $\sim 24 \mathrm{~h}$. To extract fentanyl, $5 \mathrm{~mL}$ methanol were added to each vial and mixed well. Extracts were filtered and further diluted in methanol for LC-MS analysis. A second experiment determined the recovery of fentanyl from an inactive version of SafeMedWaste I, formulated without $\mathrm{KMnO}_{4}$, TCI, or NaDCC. $2.5 \mathrm{~mL}$ of $200 \mu \mathrm{g} / \mathrm{mL}$ fentanyl were transferred to either a control vial of $2.5 \mathrm{~mL}$ water, or an aliquot of $165 \mathrm{mg}$ inactive SafeMedWaste plus $2.5 \mathrm{~mL}$ water. Vials were briefly shaken to mix the contents. After $\sim 24 \mathrm{~h}, 5 \mathrm{~mL}$ methanol were added to each vial and mixed well. The resulting extracts were filtered and further diluted for LC-MS determination of the fentanyl peak.

SafeMedWaste I beta test. The hospital BETA test was completed at a 74-bed hospital with a premier orthopedic surgery program in Columbia, SC. SafeMedWaste for the hospital beta test contained a combination of $\mathrm{KMnO}_{4}$, TCI, and NaDCC as denaturants. The controlled substance waste was collected over a 6-h period in an operating room environment. $60 \mu \mathrm{g}$ fentanyl, $26.7 \mathrm{mg}$ morphine, and $84 \mathrm{mg}$ midazolam waste were placed in the SafeMedWaste container. For testing, $700 \mathrm{~mL}$ water were slowly added to the container of SafeMedWaste and mixed well. After $\sim 24 \mathrm{~h}, 1.25 \mathrm{~g}$ of gelled waste were weighed into a vial. $20 \mathrm{~mL}$ methanol were added to the vial. The vial was capped and vortexed to mix well and extract any controlled drugs from the waste. The methanol extract was filtered with a $0.45 \mu \mathrm{m}$ RC filter into a clean vial for the analysis of morphine and midazolam. For analysis of fentanyl, the extract was concentrated tenfold by evaporating under nitrogen.

Manufacturing waste beta test. The manufacturing BETA test was completed by a 503B Outsourcing cGMP Facility that distributes billions of doses of sterile, pre-filled medications each year. This pharmaceutical manufacturer produces a variety of substances, several of which are controlled substances. 5 L containers of six separate manufacturing waste streams were received for testing with SafeMedWaste: ephedrine $5 \mathrm{mg} / \mathrm{mL}$, fentanyl $2 \mu \mathrm{g} / \mathrm{mL}$, diazepam $5 \mathrm{mg} / \mathrm{mL}$, morphine $1 \mathrm{mg} / \mathrm{mL}$, ketamine $0.6 \mathrm{mg} / \mathrm{mL}$, and hydromorphone $0.2 \mathrm{mg} / \mathrm{mL}$. Selected by the manufacturing facility, these waste streams were encompassed a wide range of drug classes. Four different formulations of SafeMedWaste were used to treat the waste samples (one formulation per drug, except for shared formulations between ephedrine and ketamine and morphine and hydromorphone). The formulations for fentanyl, morphine, and hydromorphone contained $\mathrm{KMnO}_{4}, \mathrm{NaDCC}$, and TCI as active ingredients. However, the formulation for fentanyl contained a lower quantity of active ingredients, corresponding with the overall lower concentration of fentanyl in the waste stream. The ephedrine and ketamine formulation used $\mathrm{NaDCC}$ as the active ingredient, whereas the formulation for diazepam was TCI-based.

Each $1 \mathrm{~L}$ container of SafeMedWaste received $600 \mathrm{~mL}$ of the corresponding waste sample. The waste was slowly added to the container and mixed well. After the $\sim 24$-h incubation period, aliquots containing the equivalent of $1 \mathrm{~mL}$ waste were weighed into separate glass scintillation vials. Controls were prepared by transferring 


\begin{tabular}{|c|c|c|c|c|c|}
\hline Drug class & Analyte & $\begin{array}{l}\text { Molecular weight (g/ } \\
\text { mol) }\end{array}$ & Parent mass $(\mathrm{Da})$ & Fragment mass (Da) & $\begin{array}{l}\text { Limit of detection } \\
(\mathrm{ng} / \mathrm{mL})\end{array}$ \\
\hline \multirow{7}{*}{ Opioids } & Fentanyl & 336.47 & 337.10 & 188.20 & 1 \\
\hline & Hydromorphone & 285.34 & 286.10 & 185.10 & 2 \\
\hline & Morphine & 285.34 & 286.10 & 173.00 & 5 \\
\hline & Methadone & 309.45 & 310.10 & 265.20 & 1 \\
\hline & Hydrocodone & 299.37 & 300.00 & 199.00 & 5 \\
\hline & Oxycodone & 315.37 & 316.10 & 241.10 & 1 \\
\hline & Tramadol & 263.37 & 264.30 & 58.10 & 1 \\
\hline \multirow{6}{*}{ Benzodiazepine } & Alprazolam & 308.76 & 309.10 & 205.10 & 1 \\
\hline & Chlordiazepoxide & 299.75 & 300.10 & 227.10 & 2 \\
\hline & Clonazepam & 315.715 & 316.00 & 270.20 & 1 \\
\hline & Lorazepam & 321.16 & 321.00 & 275.00 & 1 \\
\hline & Diazepam & 284.74 & 285.20 & 193.10 & 1 \\
\hline & Temazepam & 300.7 & 301.10 & 255.10 & 1 \\
\hline Non-benzodiazepine & Zolpidem & 307.40 & 308.40 & 235.10 & 1 \\
\hline \multirow{3}{*}{$\begin{array}{l}\text { Amphetamine and } \\
\text { other stimulants }\end{array}$} & Amphetamine & 135.21 & 136.10 & 90.90 & 1 \\
\hline & Ephedrine & 165.23 & 166.30 & 133.10 & 1 \\
\hline & Methylphenidate & 233.31 & 234.31 & 84.10 & 1 \\
\hline \multirow{2}{*}{ GABA analog } & Gabapentin & 171.24 & 172.24 & 137.20 & 1 \\
\hline & Pregabalin & 159.23 & 160.23 & 55.00 & 2 \\
\hline NMDA antagonist & Ketamine & 237.73 & 238.10 & 125.10 & 1 \\
\hline
\end{tabular}

Table 1. LC-MS Multiple reactions monitoring (MRM) method details. An MRM method was developed for a subset of the 34 drugs tested with SafeMedWaste, ranging across multiple classes and DEA schedules. The MRM transitions are listed, as well as the limits of detection for each analyte.

$1 \mathrm{~mL}$ of each type of waste to separate vials. 2-5 mL methanol were then added to each vial and mixed well. Extracts were filtered and further diluted in methanol for analysis. To test for solvent effects, the experiments were repeated using water, dichloromethane, and acetonitrile as extraction solvents. Ethanol and isopropyl alcohol were also tested as extraction solvents for ketamine and diazepam samples. Providing a comparison for SafeMedWaste, experiments were repeated using a charcoal-based formulation. The equivalent volume of each waste stream sample to contain $10 \mathrm{mg}$ active pharmaceutical ingredient was placed in $50 \mathrm{~g}$ of the charcoal solution, or an equivalent volume of water as control, and left for approximately $24 \mathrm{~h}$. Samples were then extracted with methanol and filtered prior to analysis.

Solid controlled medications. A representative set of medications was purchased from McKesson Medical (TX, USA): alprazolam $1 \mathrm{mg}$, amphetamine salts $30 \mathrm{mg}$, chlordiazepoxide $25 \mathrm{mg}$, clonazepam $2 \mathrm{mg}$, gabapentin $300 \mathrm{mg}$, hydrocodone/acetaminophen 10/325 mg, lorazepam $2 \mathrm{mg}$, methadone $10 \mathrm{mg}$, methylphenidate $5 \mathrm{mg}$, morphine $15 \mathrm{mg}$, oxycodone $15 \mathrm{mg}$ (in abuse-deterrent formulation), oxycodone/acetaminophen $5 / 325 \mathrm{mg}$, pregabalin $50 \mathrm{mg}$, temazepam $30 \mathrm{mg}$, tramadol $50 \mathrm{mg}$, and zolpidem $10 \mathrm{mg}$. The formulation of SafeMedWaste for the solid medications had active ingredients of $\mathrm{KMnO}_{4}$ and TCI. One unit of each medication (i.e., one tablet or one capsule) was placed either in an aliquot of SafeMedWaste, an aliquot of inactive SafeMedWaste, or in water at $\mathrm{pH} 4.00$ (adjusted with dilute hydrochloric acid) in separate plastic containers. Water was then added to the aliquots of SafeMedWaste to activate the formula. 48-72 h later, $0.5 \mathrm{~g}$ were weighed from each aliquot and extracted with methanol. Extracts were filtered, diluted in methanol, and analyzed with LC-MS. Peaks in the extracts from SafeMedWaste were compared with those in the water $\mathrm{pH} 4.00$ extract to determine denaturation. Identities of the peaks were also confirmed by analyzing external reference standards, matching on both mass and retention time. The inactive formulation of SafeMedWaste, which contains solidifying agents but no denaturants, was used to assess recovery from the solid bed matrix.

LC-MS parameters. MRM method. A multiple reactions monitoring (MRM) method was developed for alprazolam, amphetamine, butorphanol, chlordiazepoxide, clonazepam, diazepam, fentanyl, gabapentin, ketamine, lorazepam, meperidine, methadone, methamphetamine, methylphenidate, midazolam, nalbuphine, oxycodone, pregabalin, propoxyphene, remifentanil, sufentanil, tramadol and zolpidem. Analytes were chromatographed on an Agilent 1100 HPLC using a SunFire C18 $4.6 \times 150 \mathrm{~mm}, 5 \mu \mathrm{m}$ analytical column. Mobile phase A consisted of $0.1 \%$ formic acid in DI water and mobile phase B consisted of $0.1 \%$ formic acid in HPLC-grade acetonitrile. The method initial flow rate was $0.5 \mathrm{~mL} / \mathrm{min}$ at $90 \%$ mobile phase A, increasing to $1.0 \mathrm{~mL} / \mathrm{min}$ and $10 \%$ mobile phase A at $10 \mathrm{~min}$. This gradient was held for another $5 \mathrm{~min}$ until $15.0 \mathrm{~min}$ total run time, before returning to $0.5 \mathrm{~mL} / \mathrm{min}$ and $90 \%$ mobile phase A by $15.5 \mathrm{~min}$. This final gradient was held until the end of the 17.5 -min run time. The injection volume was $10 \mu \mathrm{L}$ and the column oven temperature was $30^{\circ} \mathrm{C}$. Masses corresponding to programmed parent and daughter ions for each drug (Table 1) were detected using an $\mathrm{AB}$ Sciex API 3000 triple-quadrupole mass spectrometer in positive ionization mode. 
For use in experiments with the solid controlled medications, the MRM method was adapted for a shorter run-time using a Zorbax XDB-C18 $50 \mathrm{~mm} \times 4.6 \mathrm{~mm} 1.8 \mu \mathrm{m}$ analytical column (Agilent) with the same mobile phases, column temperature, and injection volume as the longer method. Hydromorphone, morphine, and hydrocodone were also added as analytes in this method (Table 1). The flow rate was $0.6 \mathrm{~mL} / \mathrm{min}$. The initial gradient was $95 \%$ mobile phase A, decreasing to $5 \%$ mobile phase A over 4 min. The gradient was held at $5 \%$ mobile phase A until 4.5 min run-time before returning to $95 \%$ mobile phase A by $5.0 \mathrm{~min}$. This gradient was held until the end of the method. Method limits of detection were established using analytical reference standards prepared in methanol, demonstrating that all 20 drugs were detectable at $5 \mathrm{ng} / \mathrm{mL}$ or lower (Table 1).

Scan method. A scan-mode LC-MS method with positive ionization was also developed. This method demonstrated that drugs were fully destroyed in SafeMedWaste, as masses corresponding to active metabolites or precursors were scanned and not found. Mobile phases, injection volume, and the analytical column were identical to those in the 17.5-min MRM method. The method flow rate was $1.0 \mathrm{~mL} / \mathrm{min}$. The first $5 \mathrm{~min}$ of the method held at $95 \%$ mobile phase A, decreasing to $1 \%$ mobile phase A at $20 \mathrm{~min}$. This gradient was held until $25.0 \mathrm{~min}$ total run time, before returning to $95 \%$ mobile phase A by $25.1 \mathrm{~min}$. This final gradient was held until the end of the run-time, $30 \mathrm{~min}$. The column oven temperature was uncontrolled. Using an AB Sciex API 3000 triplequadrupole mass spectrometer in positive ionization mode, masses from 50-1500 Da were scanned throughout the method. In contrast, pentobarbital and phenobarbital were detected using negative ESI. Propofol was poorly resolved by MS, so instead a diode array detector was used to detect propofol at a wavelength of $270 \mathrm{~nm}$.

\section{Results}

Development of four formulations of SafeMedWaste to destroy $\mathbf{3 4}$ drugs. To denature all 34 liquid drugs efficiently, four different formulations of SafeMedWaste were developed (SafeMedWaste I-IV). Representative LC-MS chromatograms of a fentanyl standard peak and of the fentanyl peak after 24-h incubation in each variation of SafeMedWaste are shown in Fig. 1. In 24 h, SafeMedWaste I active ingredient solution fully denatured 32 of the 34 drugs tested. However, the drugs denatured most efficiently in terms of ratio of SafeMedWaste to active pharmaceutical ingredient (API) in SafeMedWaste formulation I versus formulations II-IV included hydrocodone, hydromorphone, methadone, morphine, nalbuphine, oxycodone, propoxyphene, THC, and zolpidem. However, SafeMedWaste I failed to denature two drugs, cocaine and pentobarbital. For example, cocaine was only 6\% denatured (Supplemental Fig. 1A), whereas pentobarbital was 67\% denatured within $24 \mathrm{~h}$ in SafeMedWaste I.

SafeMedWaste II was the most effective denaturant for 16 of the 34 drugs on the list, fully denaturing each API within $24 \mathrm{~h}$. These drugs included amphetamine, butorphanol, clonazepam, codeine, ephedrine, fentanyl, gabapentin, ketamine, meperidine, methamphetamine, methylphenidate, pentobarbital, phenobarbital, pregabalin, propofol, and sufentanil. SafeMedWaste III was the most effective formulation for eight of the 34 drugs: alprazolam, chlordiazepoxide, diazepam, lorazepam, midazolam, remifentanil, temazepam, and tramadol. Cocaine received its own formulation of SafeMedWaste, SafeMedWaste IV (Supplemental Fig. 1B). This was the only formulation that successfully denatured cocaine in $24 \mathrm{~h}$. Drugs denatured in each of the four formulations of SafeMedWaste and the ratio of grams active ingredients per gram API to ensure full denaturation are summarized in Table 2. Demonstrating that the addition of solidifiers to SafeMedWaste active ingredients did not prevent the denaturation of the API, $200 \mu \mathrm{g} / \mathrm{mL}$ fentanyl was successfully denatured in SafeMedWaste I plus the addition of solidifiers within $24 \mathrm{~h}$ (Supplemental Fig. 2A). Recovery of fentanyl from the solidifying ingredients of SafeMedWaste was $83 \%$ after $24 \mathrm{~h}$ (Supplemental Fig. 2B).

Results of beta test in hospital setting. A beta test sample of SafeMedWaste I was collected in a clinical setting (Supplemental Table 1). Based on the log sheet filled out by nurses participating in the study, the extract from SafeMedWaste I beta test sample contained approximately $4.3 \mathrm{ng} / \mathrm{mL}$ fentanyl, $6.0 \mu \mathrm{g} / \mathrm{mL}$ midazolam, and $1.9 \mu \mathrm{g} / \mathrm{mL}$ morphine. The $10 \times$ concentrated extract contained approximately $43 \mathrm{ng} / \mathrm{mL}$ fentanyl, $60 \mu \mathrm{g} / \mathrm{mL}$ midazolam, and $19 \mu \mathrm{g} / \mathrm{mL}$ morphine. Both extracts were analyzed with LC-MS using the scan mode method, finding that all three of the controlled drugs had been fully denatured to the extent that they were no longer quantifiable (i.e. below the method limits of quantitation of $10 \mathrm{ng} / \mathrm{mL}$ for fentanyl and midazolam and $20 \mathrm{ng} /$ $\mathrm{mL}$ for morphine). Supplemental Fig. 3 contains chromatograms showing reference standards of fentanyl, midazolam, and morphine, and of the SafeMedWaste I beta test sample.

Results of beta test of manufacturing waste stream samples. The API in each of the six waste streams was fully denatured in each formulation of SafeMedWaste (Table 3). Each $1 \mathrm{~L}$ container, which could denature the API from $600 \mathrm{~mL}$ of each waste stream, contained approximately 130-150 g of SafeMedWaste with varying concentrations of active ingredients. Based on the concentrations of API in each waste stream, ratios of active ingredients needed per gram API in the waste stream were calculated and are shown in Table 3. For morphine and hydromorphone waste streams, a 72 -h incubation time was required for denaturation $<98 \%$. Confirming that the effect was not specific to the extraction solvent, various extraction solvents were used in place of methanol, all producing the same result: API was fully denatured in each formulation of SafeMedWaste. Chromatograms showing the diazepam waste stream before and after treatment with SafeMedWaste are shown in Fig. 2, and chromatograms from the other five waste streams are shown in Supplemental Fig. 4A-E.

Comparison between SafeMedWaste and charcoal-based alternative. A charcoal-based formulation, which claims to destroy controlled substance waste at a ratio of $50 \mathrm{~g}$ per $10 \mathrm{mg}$ API, was also tested with the six waste stream samples. In contrast to samples treated with SafeMedWaste, API was recovered from 


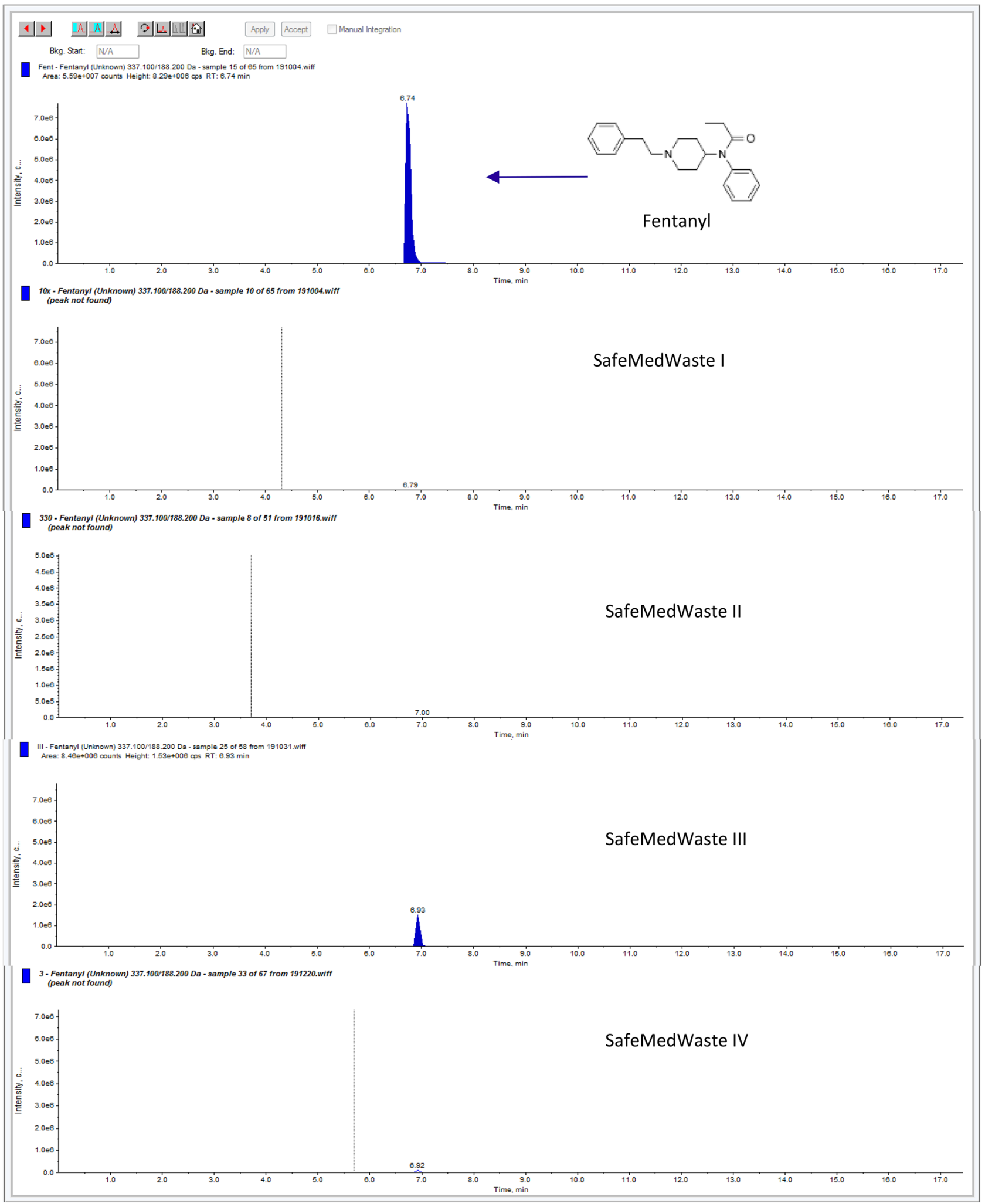

Figure 1. Denaturing fentanyl in SafeMedWaste. This figure shows LC-MS chromatograms of the fentanyl peak in control sample (top panel) and after SafeMedWaste I-IV (second from top to bottom panel). The fentanyl peak remained above the detection limit of $1 \mathrm{ng} / \mathrm{mL}$ in SafeMedWaste III but was denatured in the other three formulations. 


\begin{tabular}{|c|c|c|c|c|c|c|c|c|c|}
\hline \multirow{2}{*}{ Drug } & \multirow{2}{*}{$\begin{array}{c}\text { DEA } \\
\text { Schedule }\end{array}$} & \multicolumn{2}{|c|}{ SafeMedWaste I } & \multicolumn{2}{|c|}{ SafeMedWaste II } & \multicolumn{2}{|c|}{ SafeMedWaste III } & \multicolumn{2}{|c|}{ SafeMedWaste IV } \\
\hline & & Denatured? & Ratio & Denatured? & Ratio & Denatured? & Ratio & Denatured? & Ratio \\
\hline Alprazolam & IV & $\mathrm{Y}$ & 99 & $\mathrm{~N}$ & N/A & $Y$ & \begin{tabular}{|l|}
9.9 \\
\end{tabular} & $\mathrm{~N}$ & N/A \\
\hline Amphetamine & II & $Y$ & 19.8 & $Y$ & 9.9 & N & N/A & $\mathrm{N}$ & N/A \\
\hline Butorphanol & IV & $Y$ & 1.98 & $Y$ & 3.3 & $Y$ & 9.9 & $Y$ & 3.3 \\
\hline Chlordiazepoxide & IV & $Y$ & 99 & $\mathrm{~N}$ & $\mathrm{~N} / \mathrm{A}$ & $Y$ & 19.8 & $\mathrm{~N}$ & N/A \\
\hline Clonazepam & IV & $Y$ & 9.9 & $Y$ & 3.3 & $\mathrm{~N}$ & $\mathrm{~N} / \mathrm{A}$ & $\mathrm{N}$ & N/A \\
\hline Cocaine & II & $\mathrm{N}$ & $\mathrm{N} / \mathrm{A}$ & $\mathrm{N}$ & $\mathrm{N} / \mathrm{A}$ & $\mathrm{N}$ & N/A & $Y$ & 50 \\
\hline Codeine & II & $Y$ & 1.98 & $Y$ & 3.3 & $Y$ & 3.3 & $Y$ & 3.3 \\
\hline Diazepam & IV & $Y$ & 99 & $\mathrm{~N}$ & N/A & $Y$ & 9.9 & $\mathrm{~N}$ & N/A \\
\hline Ephedrine & $\mathrm{N} / \mathrm{A}$ & $Y$ & 9.9 & $Y$ & 9.9 & $\mathrm{~N}$ & N/A & $\mathrm{Y}$ & 3.3 \\
\hline Fentanyl & $\mathrm{II}$ & $Y$ & 9.9 & $Y$ & 3.3 & $\mathrm{~N}$ & N/A & $Y$ & 3.3 \\
\hline Gabapentin & $\mathrm{N} / \mathrm{A}$ & $Y$ & 9.9 & $Y$ & 3.3 & $Y$ & 3.3 & $\mathrm{~N}$ & N/A \\
\hline Hydrocodone & II & $Y$ & 2.64 & $Y$ & 9.9 & $Y$ & 9.9 & $Y$ & 9.9 \\
\hline Hydromorphone & II & $Y$ & 1.32 & $Y$ & 3.3 & $Y$ & 3.3 & $Y$ & 3.3 \\
\hline Ketamine & III & $Y$ & 99 & $Y$ & 9.9 & $\mathrm{~N}$ & $\mathrm{~N} / \mathrm{A}$ & $\mathrm{N}$ & N/A \\
\hline Lorazepam & IV & $Y$ & 9.9 & $Y$ & 3.3 & $Y$ & 3.3 & $Y$ & 39.6 \\
\hline Meperidine & II & $Y$ & 9.9 & $Y$ & 9.9 & $\mathrm{~N}$ & N/A & $\mathrm{N}$ & N/A \\
\hline Methadone & II & $Y$ & 2.64 & $\mathrm{~N}$ & $\mathrm{~N} / \mathrm{A}$ & $\mathrm{N}$ & N/A & $Y$ & 3.3 \\
\hline Methamphetamine & II & $Y$ & 99 & $Y$ & 40 & $\mathrm{~N}$ & N/A & $\mathrm{N}$ & N/A \\
\hline Methylphenidate & II & $Y$ & 9.9 & $Y$ & 3.3 & $Y$ & 9.9 & $Y$ & 19.8 \\
\hline Midazolam & IV & $Y$ & 99 & $Y$ & 40 & $Y$ & 9.9 & $Y$ & 39.6 \\
\hline Morphine & II & $Y$ & 1.32 & $Y$ & 3.3 & $Y$ & 3.3 & $Y$ & 3.3 \\
\hline Nalbuphine & II & $Y$ & 0.99 & $Y$ & 3.3 & $Y$ & 3.3 & $Y$ & 3.3 \\
\hline Oxycodone & II & $Y$ & 2.64 & $Y$ & 40 & $Y$ & 19.8 & $Y$ & 3.3 \\
\hline Pentobarbital & II & $\mathrm{N}$ & $\mathrm{N} / \mathrm{A}$ & $Y$ & 40 & $\mathrm{~N}$ & $\mathrm{~N} / \mathrm{A}$ & $\mathrm{N}$ & N/A \\
\hline Phenobarbital & IV & $Y$ & 99 & $Y$ & 9.9 & $\mathrm{~N}$ & $\mathrm{~N} / \mathrm{A}$ & $\mathrm{N}$ & N/A \\
\hline Pregabalin & $\mathrm{V}$ & $Y$ & 9.9 & $Y$ & 3.3 & $Y$ & 3.3 & $\mathrm{~N}$ & N/A \\
\hline Propofol & IV & $Y$ & 1.98 & $Y$ & 3.3 & $Y$ & 3.3 & $Y$ & 3.3 \\
\hline Propoxyphene & II & $Y$ & 4.95 & $\mathrm{~N}$ & N/A & $\mathrm{N}$ & N/A & $Y$ & 3.3 \\
\hline Remifentanil & II & $Y$ & 19.8 & $Y$ & 40 & $Y$ & 9.9 & $Y$ & 19.8 \\
\hline Sufentanil & II & $Y$ & 9.9 & $Y$ & 9.9 & $Y$ & 9.9 & Y & 19.8 \\
\hline $\mathrm{THC}$ & 1 & $Y$ & 0.99 & $Y$ & 40 & $Y$ & 3.3 & $Y$ & 3.3 \\
\hline Temazepam & IV & $\mathrm{N}$ & $\mathrm{N} / \mathrm{A}$ & $Y$ & 40 & $Y$ & 19.8 & $Y$ & 39.6 \\
\hline Tramadol & IV & $Y$ & 9.9 & $Y$ & 9.9 & $Y$ & 9.9 & $Y$ & 3.3 \\
\hline Zolpidem & IV & $Y$ & 0.99 & $Y$ & 3.3 & $Y$ & 3.3 & $Y$ & 19.8 \\
\hline
\end{tabular}

Table 2. Compatibility of 34 drugs with SafeMedWaste formulations I-IV. Y=yes, $N=$ no. Cells highlighted yellow indicate that formulation of SafeMedWaste is the most efficient formulation (in terms of both efficacy and cost) for that drug. Cells highlighted red indicate incompatibility, in that the drug was not fully denatured in the indicated formulation of SafeMedWaste. The ratios are the amount of SafeMedWaste active ingredients (in grams) required to denature $1 \mathrm{~g}$ of controlled substance.

samples treated with activated charcoal using methanol as an extraction solvent (Table 4). The effect was drugdependent, with ketamine and morphine showing no denaturation after $24 \mathrm{~h}$ and with diazepam showing over $90 \%$ denaturation in the same timeframe (Supplemental Fig. 5). Figure 3 illustrates the peak areas in extracts from activated charcoal samples versus control samples in a bar graph format. When water was tested as an extraction solvent, recovery of controlled substances from the charcoal matrix was poor, indicating that they had been adsorbed to the charcoal surface. On the surface, a lack of a peak in the water extract may make it appear that the drug has been denatured. However, results from the methanol extract demonstrated that drugs were not chemically altered after adsorption, as they could be successfully recovered from the matrix. Additional products sold as disposal systems for controlled medications also failed to denature fentanyl, ketamine, and morphine 


\begin{tabular}{|c|c|c|c|c|c|}
\hline Drug & Structure & $\begin{array}{l}\text { Concentration in } \\
\text { waste }\end{array}$ & Ratio per gram API & Extraction solvent & $\begin{array}{l}\text { \% Denatured by } \\
\text { LC-MS }\end{array}$ \\
\hline \multirow{6}{*}{ Diazepam } & & \multirow{6}{*}{$5 \mathrm{mg} / \mathrm{mL}$} & \multirow{6}{*}{$8.3 \mathrm{~g}$} & $\mathrm{MeOH}$ & Fully \\
\hline & & & & ACN & Fully \\
\hline & & & & Water & Fully \\
\hline & & & & DCM & Fully \\
\hline & & & & EtOH & Fully \\
\hline & & & & IPA & Fully \\
\hline \multirow{4}{*}{ Ephedrine } & & \multirow{4}{*}{$5 \mathrm{mg} / \mathrm{mL}$} & \multirow{4}{*}{$13.3 \mathrm{~g}$} & $\mathrm{MeOH}$ & Fully \\
\hline & & & & $\mathrm{ACN}$ & Fully \\
\hline & & & & Water & Fully \\
\hline & & & & DCM & Fully \\
\hline \multirow{4}{*}{ Fentanyl } & & \multirow{4}{*}{$2 \mu \mathrm{g} / \mathrm{mL}$} & \multirow{4}{*}{$6.75 \mathrm{~g}$ per $\mathrm{mg}$} & $\mathrm{MeOH}$ & Fully \\
\hline & & & & ACN & Fully \\
\hline & & & & Water & Fully \\
\hline & & & & DCM & Fully \\
\hline \multirow{4}{*}{ Hydromorphone } & & \multirow{4}{*}{$0.2 \mathrm{mg} / \mathrm{mL}$} & \multirow{4}{*}{$40 \mathrm{~g}$} & $\mathrm{MeOH}$ & Fully \\
\hline & & & & $\mathrm{ACN}$ & Fully \\
\hline & & & & Water & Fully \\
\hline & & & & DCM & Fully \\
\hline \multirow{6}{*}{ Ketamine } & & \multirow{6}{*}{$0.6 \mathrm{mg} / \mathrm{mL}$} & \multirow{6}{*}{$69.4 \mathrm{~g}$} & $\mathrm{MeOH}$ & Fully \\
\hline & & & & ACN & Fully \\
\hline & & & & Water & Fully \\
\hline & & & & DCM & Fully \\
\hline & & & & EtOH & Fully \\
\hline & & & & IPA & Fully \\
\hline \multirow{4}{*}{ Morphine } & & \multirow{4}{*}{$1 \mathrm{mg} / \mathrm{mL}$} & \multirow{4}{*}{$8 \mathrm{~g}$} & $\mathrm{MeOH}$ & Fully \\
\hline & & & & $\mathrm{ACN}$ & Fully \\
\hline & & & & Water & Fully \\
\hline & & & & DCM & Fully \\
\hline
\end{tabular}

Table 3. Summary of beta test using manufacturing waste stream. The ratio is the grams of SafeMedWaste active ingredients required to denature $1 \mathrm{~g}$ of $\mathrm{API}$ in the waste stream. $\mathrm{MeOH}=$ methanol, $\mathrm{ACN}=$ acetonitrile, $\mathrm{DCM}=$ dichloromethane, $\mathrm{EtOH}=$ ethanol, $\mathrm{IPA}=$ isopropyl alcohol. 


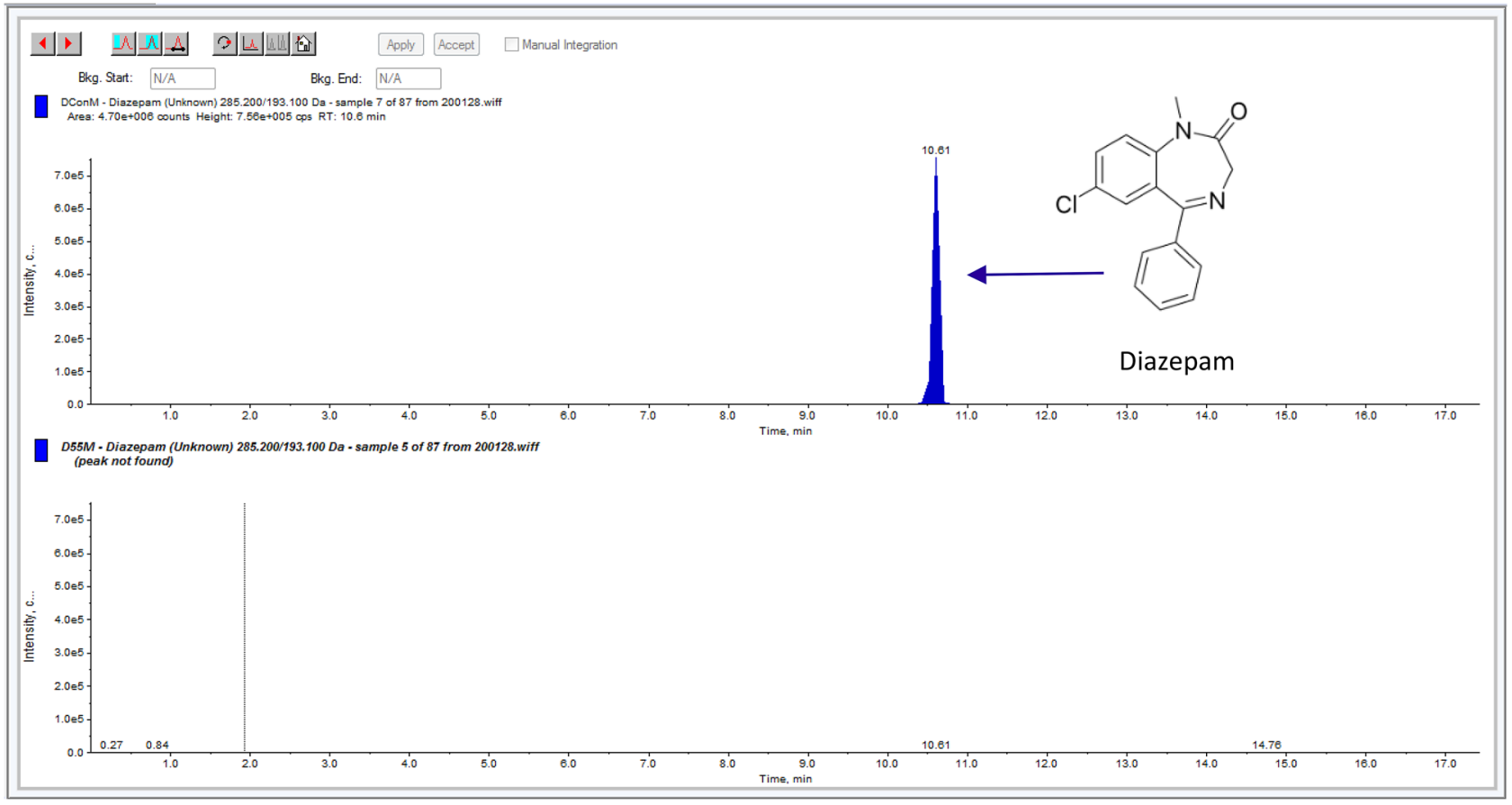

Figure 2. SafeMedWaste denatures diazepam waste generated by a manufacturing facility. An LC-MS chromatogram of the $5 \mathrm{mg} / \mathrm{mL}$ diazepam waste stream sample before treatment with SafeMedWaste is shown in the top panel, whereas a chromatogram of the same waste sample after treatment with SafeMedWaste is shown in the bottom panel. After treatment with SafeMedWaste, the diazepam peak was below the limit of detection of $1 \mathrm{ng} / \mathrm{mL}$.

when tested in the same manner as the charcoal-based option. While the ingredients of these products were not disclosed, the recovery of fentanyl, ketamine, and morphine in the methanol extract suggested that these products were also reliant on adsorption rather than chemical degradation.

Results of experiments with solid controlled medications. A single variation of SafeMedWaste was created for denaturing solid controlled medications, such as capsules and tablets. Each medication tested was fully denatured in varying concentrations of this formulation within $72 \mathrm{~h}$ (Table 5). It was found that drugs containing acetaminophen tended to require more SafeMedWaste, possibly as the acetaminophen competes for the active ingredients. However, in general, more SafeMedWaste was required for solid medications than for liquid medications. Medications were also tested on an inactive control formulation of SafeMedWaste to assess the recovery from the solidifier matrix. The concentrations of drug in the extracts from the active formulations of SafeMedWaste were then corrected for percent recovery to account for matrix effects. Recoveries tended to be lower for drugs formulated as extended release capsules and for tablets containing acetaminophen. Representative chromatograms of the controlled API before and after treatment with SafeMedWaste, both inactive and active, are shown in Supplemental Fig. 6.

\section{Discussion}

Results demonstrate that SafeMedWaste is an effective chemical denaturant of a wide range of controlled medications, and presents a safe and effective solution to the issue of controlled substance waste disposal. While other products have focused on single categories of drugs, such as benzodiazepines ${ }^{21}$, a larger subset of drugs was chosen for these studies based on personal communications with hospitals, law enforcement, and pharmacies. However, it was found that the efficacy of SafeMedWaste varies by drug type, such that four different formulations were developed for the maximum efficiency. Each of the formulations follows the same basic format: a combination of denaturants, which primarily degrade the API via oxidation, and solidifying agents that eventually absorb the water added to activate SafeMedWaste and turn the entire formulation into a solid for easy disposal. The primary formulation of SafeMedWaste for liquid medications is versatile, with only pentobarbital and cocaine failing to degrade in this mixture. However, by customizing the formulation of active ingredients of SafeMedWaste, effective denaturants for pentobarbital and cocaine were identified. Beta testing of SafeMedWaste 


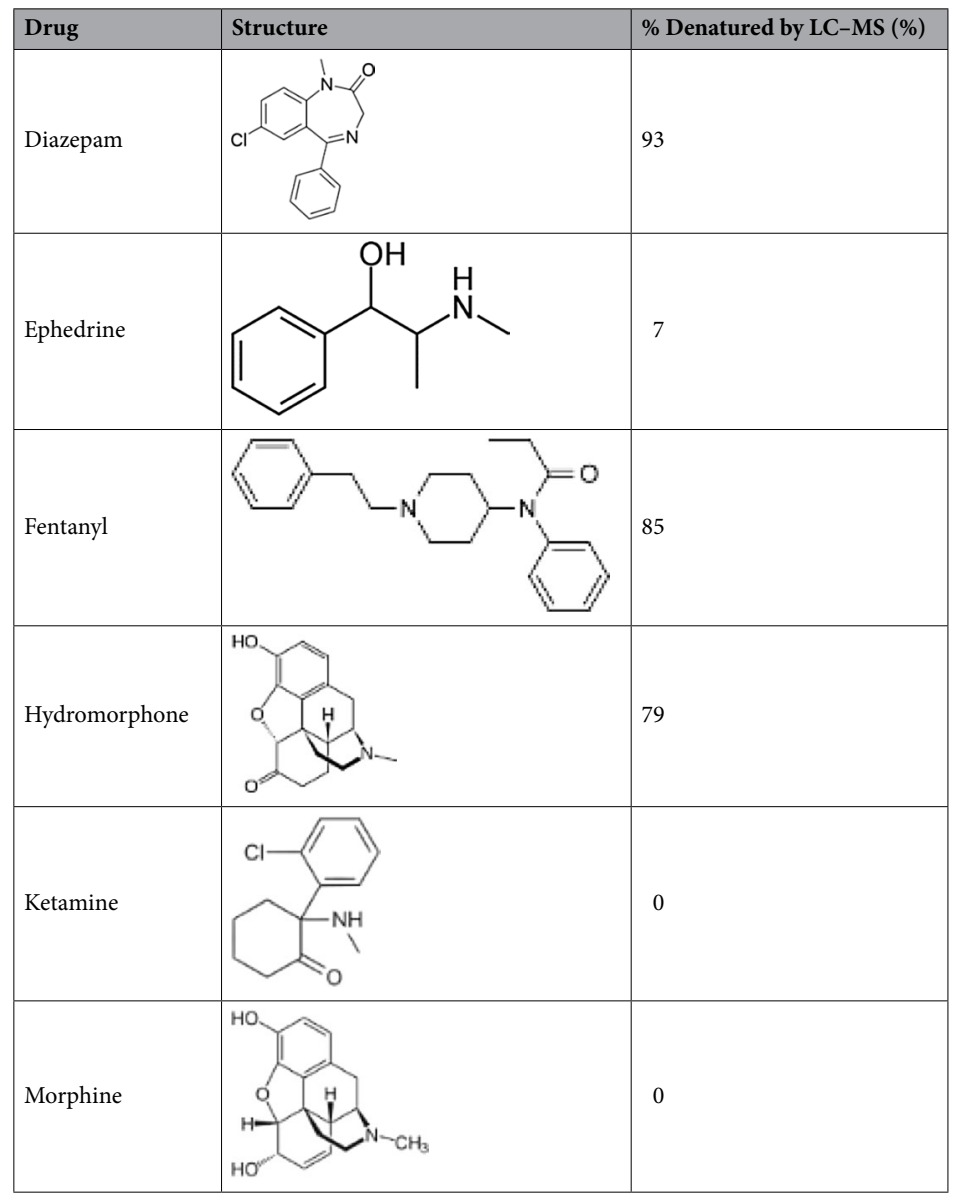

Table 4. Results for waste stream samples treated with activated charcoal. Waste stream samples were treated with water or activated charcoal for $24 \mathrm{~h}$. Medications in the samples were extracted with methanol and analyzed with LC-MS. Peak areas were compared between control (water) and experimental (activated charcoal) conditions to calculate the \% denatured. Denaturation was drug-specific; ketamine and morphine showed full recovery from activated charcoal, whereas diazepam was over $90 \%$ denatured.

was carried out in two different settings: a manufacturing facility and a hospital. In both cases, SafeMedWaste was an effective means of removing traces of controlled medications.

SafeMedWaste is not the only product for disposal controlled medications on the market ${ }^{22}$. Other products often use charcoal as their active ingredient ${ }^{21,23}$, of which is an attractive choice due to a low cost and low toxicity. Indeed, there is evidence that compounds such as diazepam, will adsorb to the activated charcoal surface and can not be recovered from activated charcoal using water or $30 \%$ ethanol after 28 days ${ }^{21}$. Another charcoalbased system showed similar results for lorazepam, diazepam, and buprenorphine using HPLC ${ }^{23}$. However, it was shown in the present studies that these compounds can be extracted from charcoal in solvents such as methanol, ethanol, acetonitrile, and isopropyl alcohol. The extraction process is not complex and can be done with household chemicals, underscoring the need for a product that can chemically alter controlled substances to inactivate them, rather than simply inactivate them via sequestration. Our data also illustrated that diazepam may be the most readily adsorbed to a charcoal matrix, with only $7 \%$ recovery after $24 \mathrm{~h}$ in a methanol extraction. For other drugs with limited disposal options, such as ketamine $e^{24,25}$, the recovery from the charcoal matrix was close to $100 \%$.

A formulation of SafeMedWaste for solid medications was designed with promising results: full denaturation of 15 different solid medications was demonstrated via LC-MS in 48-72 h. These drugs included time-release formulations of amphetamine salts and morphine, which contain complex excipients to slowly release the API ${ }^{26,27}$. Several benzodiazepines were included as well, as these represent a commonly prescribed class of medications with abuse liability ${ }^{28-30}$. While SafeMedWaste has been tested on 15 different solid medications, more testing 


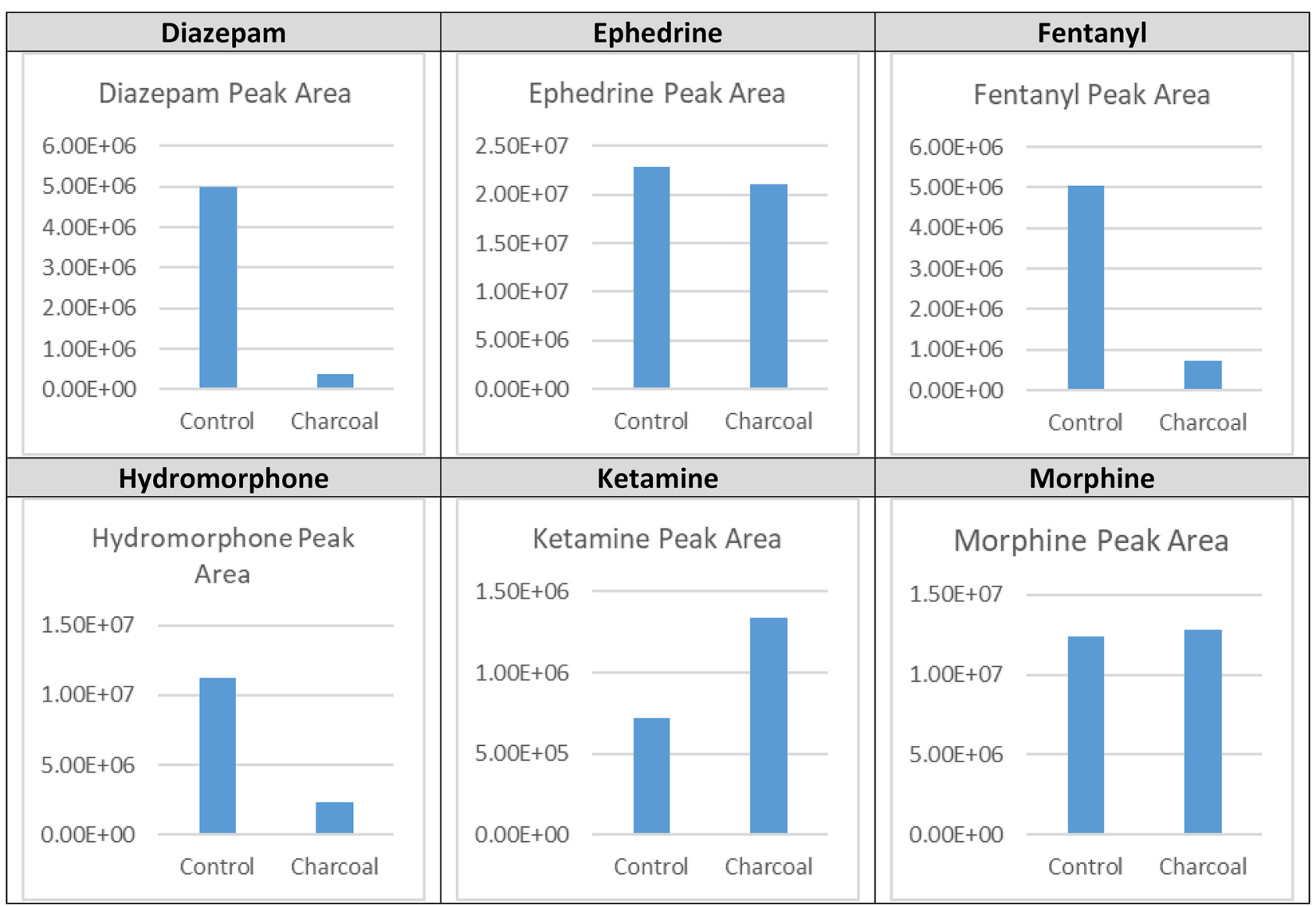

Figure 3. Controlled substances are retrievable from activated charcoal-based products. Bar graphs represent the peak areas in waste stream samples treated with activated charcoal solution versus control. Waste stream samples were diazepam $5 \mathrm{mg} / \mathrm{mL}$, ephedrine $5 \mathrm{mg} / \mathrm{mL}$, fentanyl $2 \mu \mathrm{g} / \mathrm{mL}$, hydromorphone $0.2 \mathrm{mg} / \mathrm{mL}$, ketamine $0.6 \mathrm{mg} / \mathrm{mL}$, and morphine $1 \mathrm{mg} / \mathrm{mL}$.

will be needed to confirm efficacy on a wider subset of drugs. Given that the 15 medications tested covered a wide range of drug classes, it is predicted that SafeMedWaste will be applicable to more medications. A follow-up study will aim to beta test the formulation of SafeMedWaste for solid medications in a pharmacy and residential setting. Participants would receive a disposal kit of SafeMedWaste (at no cost) when they fill their prescription. If needed, they can use it to dispose of any unused portion, which will be collected for LC-MS analysis to confirm whether the medictations were destroyed.

Previous research has shown that patients are uncomfortable with the idea of flushing unused medication ${ }^{31}$ and respond positively to the inclusion of a disposal bag with their prescriptions: the rate of proper disposal of opioid medications increased approximately $20 \%$ amongst families of children receving postoperative opioids when the disposal bag was included ${ }^{7}$. In that study, the efficacy of the disposal bag was not reported. However, their data suggests that patients respond well to being given a safe and easy option to dispose of their unused medication. A survey of 152 patients who were in possession of unused prescription opioid medications found that over $80 \%$ of participants agreed that they would be more likely to use a drug take-back program if it were offered in a convenient location, such as a pharmacy ${ }^{32}$. Another study showed that, while around $50 \%$ of prescribed opioids went unused after dental surgery, the likelihood of patients to dispose of the unused medications increased $22 \%$ when they were informed of a pharmacy take-back program ${ }^{8}$. Taken together, these findings demonstrate that many patients do not want to contribute to the problem of diversion of unused medications, but that effective in-home disposal options are very limited.

\section{Conclusions}

There is a need for safe, effective products to destroy controlled substance waste in the factory, in the clinic, and in the home. SafeMedWaste, a patented blend of chemical denaturants and solidifying agents, was shown to fully denature 34 liquid controlled medications and 15 solid controlled medications using LC-MS. Efficacy was also demonstrated on controlled substance waste collected in a hospital and in a pharmaceutical manufacturing facility. In conclusion, SafeMedWaste destroys both liquid and solid controlled medications and represents a novel, yet practical, approach to reducing drug diversion. 


\begin{tabular}{|c|c|c|c|c|c|}
\hline Drug & Structure & Dose (mg) & SafeMedWaste (mg) & Active ingredients (mg) & $\begin{array}{l}\text { Ratio of } g \text { active ingredients } \\
\text { per } 1 \mathrm{~g} \text { API }\end{array}$ \\
\hline Alprazolam & & 1 & 180 & 60 & 60:1 \\
\hline $\begin{array}{l}\text { Amphetamine salts (extended } \\
\text { release) }\end{array}$ & & 30 & 1500 & 500 & $20: 1$ \\
\hline Chlordiazepoxide & & 25 & 500 & 167 & $7: 1$ \\
\hline Clonazepam & & 2 & 250 & 83 & $42: 1$ \\
\hline Gabapentin & & 300 & 4000 & 1333 & $5: 1$ \\
\hline Hydrocodone & & 10 (plus $325 \mathrm{mg}$ acetaminophen) & 1000 & 333 & $33: 1$ \\
\hline Lorazepam & & 2 & 300 & 100 & $50: 1$ \\
\hline Methadone & & 10 & 420 & 140 & $14: 1$ \\
\hline Methylphenidate & & 5 & 250 & 83 & $17: 1$ \\
\hline Morphine & & 15 & 225 & 75 & $5: 1$ \\
\hline Oxycodone & & 5 (plus $325 \mathrm{mg}$ acetaminophen) & 1000 & 333 & $67: 1$ \\
\hline Pregabalin & & 50 & 750 & 250 & $5: 1$ \\
\hline Temazepam & & 30 & 450 & 150 & 5:1 \\
\hline Tramadol & & 50 & 333 & 111 & $2: 1$ \\
\hline Zolpidem & & 10 & 300 & 100 & $10: 1$ \\
\hline
\end{tabular}

Table 5. Summary of experiments on solid controlled medications. The formulation of SafeMedWaste for solid medications consisted of 1-part active denaturants to 2-parts solidifiers. Tablets/capsules were treated with SafeMedWaste or a control solution (water $\mathrm{pH} 4.00$ ) for $48-72 \mathrm{~h}$. Samples were extracted with methanol and analyzed with LC-MS. 
Received: 30 September 2020; Accepted: 21 December 2020

Published online: 13 January 2021

\section{References}

1. Kochanek, K. D., Murphy, S. L., Xu, J. \& Arias, E. Deaths: final data for 2017. Natl. Vital Stat. Rep. 68, 1-77 (2019).

2. Lipari, R. N. \& Van Horn, S. L. Trends in substance use disorders among adults aged 18 or older (2017).

3. Koob, G. F. \& Volkow, N. D. Neurobiology of addiction: a neurocircuitry analysis. Lancet Psychiatry 3, 760-773. https://doi. org/10.1016/S2215-0366(16)00104-8 (2016).

4. Valentino, R. J., Koroshetz, W. \& Volkow, N. D. Neurobiology of the opioid epidemic: basic and translational perspectives. Biol. Psychiatry 87, 2-3 (2020).

5. Brat, G. A. et al. Postsurgical prescriptions for opioid naive patients and association with overdose and misuse: retrospective cohort study. BMJ 360, j5790. https://doi.org/10.1136/bmj.j5790 (2018).

6. Murthy, V. H. Ending the opioid epidemic - a call to action. N. Engl. J. Med. 375, 2413-2415 (2016).

7. Lawrence, A. E. et al. Effect of drug disposal bag provision on proper disposal of unused opioids by families of pediatric surgical patients: a randomized clinical trial. JAMA Pediatr. 173, e191695. https://doi.org/10.1001/jamapediatrics.2019.1695 (2019).

8. Maughan, B. C. et al. Unused opioid analgesics and drug disposal following outpatient dental surgery: a randomized controlled trial. Drug Alcohol Depend. 168, 328-334 (2016).

9. Akici, A., Aydin, V. \& Kiroglu, A. Assessment of the association between drug disposal practices and drug use and storage behaviors. Saudi Pharmaceut. J. 26, 7-13 (2018).

10. Gregorian, R. et al. Safe opioid storage and disposal: a survey of patient beliefs and practices. J. Pain Res. 13, 987-995. https://doi. org/10.2147/JPR.S242825 (2020).

11. Lipari, R. N. \& Hughes, A. in The CBHSQ report (Substance Abuse and Mental Health Services Administration (US), 2017).

12. Owens, B. Pharmaceuticals in the environment: a growing problem. The Pharmaceutical Journal (2015). https://www.pharmaceut ical-journal.com/news-and-analysis/features/pharmaceuticals-in-the-environment-a-growing-problem/.

13. Ramos, H. M. P. et al. Medication disposal: a reflection about possible sanitary and environmental risks. Ambiente Sociedade 20, 145-168 (2017).

14. Kinrys, G., Gold, A. K., Worthington, J. J. \& Nierenberg, A. A. Medication disposal practices: increasing patient and clinician education on safe methods. J. Int. Med. Res. 46, 927-939. https://doi.org/10.1177/0300060517738681 (2018).

15. Lindsay, E. The High Cost of Drug Diversion. The Pharmacy Times (2016). https://www.pharmacytimes.com/contributor/erica -lindsay-pharmd-mba-jd/2016/01/the-high-cost-of-drug-diversion.

16. Kenna, G. A. \& Lewis, D. C. Risk factors for alcohol and other drug use by healthcare professionals. Subst. Abuse Treat. Prev. Policy 3, 3 (2008).

17. Perry, J. C. \& Vandenhouten, C. L. Drug diversion detection. Nurs. Manag. 50, 16-21. https://doi.org/10.1097/01.NUMA.00005 52735.56577.4b (2019)

18. Title 21 Code of Federal Regulations, Part 1317 Subpart C-Destruction of Controlled Substances. DEA Diversion Control Division (2020). https://www.deadiversion.usdoj.gov/21cfr/cfr/1317/subpart_c.htm.

19. Brechtelsbauer, E. \& Shah, S. Update on pharmaceutical waste disposal regulations: strategies for success. Am. J. Health Syst. Pharm. 77, 574-582 (2020).

20. Stas, J., Wang, Y., Boyst, E., Channu, V. S. R. \& Leishman, E. United States patent 10668312 (2020).

21. Dasht Bozorg, B., Fowler, W., Korey, A., Anderson, C. \& Banga, A. K. Evaluation of an activated carbon disposal system for safe disposal of model prescription sedative medications. Sci. Rep. 10, 2968. https://doi.org/10.1038/s41598-020-59907-2 (2020).

22. Imarhia, F., Varisco, T. J., Wanat, M. A. \& Thornton, J. D. Prescription drug disposal: products available for home use. J. Am. Pharm. Assoc. 60, e7-e13 (2020).

23. Song, Y., Manian, M., Fowler, W., Korey, A. \& Kumar Banga, A. Activated carbon-based system for the disposal of psychoactive medications. Pharmaceutics https://doi.org/10.3390/pharmaceutics8040031 (2016).

24. Pleva, A. \& Davis, R. G. Legal considerations of ketamine for veterinary practices. Plumb's Therapeutics Brief 62-64 (2017).

25. Zhang, H. F., Yang, J., Du, P., Wang, C. C. \& Li, X. Q. Concentrations of ketamine and norketamine in the water environment in Beijing. Huan jing ke xue = Huanjing kexue 37, 2522-2529. https://doi.org/10.13227/j.hjkx.2016.07.014 (2016)

26. Khatri, P., Desai, D., Shelke, N. \& Minko, T. Role of plasticizer in membrane coated extended release oral drug delivery system. J. Drug Deliv. Sci. Technol. 44, 231-243 (2018).

27. Murugesan, S., Gowramma, B., Lakshmanan, K., Reddy Karri, V. V. S. \& Radhakrishnan, A. Oral modified drug release solid dosage form with special reference to design; an overview. Curr. Drug Res. Rev. 12, 16-25. https://doi.org/10.2174/258997751166619 1121094520 (2020).

28. McHugh, R. K., Peckham, A. D., Björgvinsson, T., Korte, F. M. \& Beard, C. Benzodiazepine misuse among adults receiving psychiatric treatment. J. Psychiatr. Res. 128, 33-37 (2020).

29. Knopf, A. FDA orders addition to boxed warning for benzodiazepines. Alcoho. Drug Abuse Weekly 32, 3-5 (2020).

30. Schmitz, A. Benzodiazepine use, misuse, and abuse: a review. Mental Health Clin. 6, 120-126 (2016).

31. White, C. A. \& Triboletti, M. D. Implementation of a patient medication disposal program at a VA medical center. Fed. Pract. 34, 45 (2017).

32. Buffington, D. E., Lozicki, A., Alfieri, T. \& Bond, T. C. Understanding factors that contribute to the disposal of unused opioid medication. J. Pain Res. 12, 725 (2019).

\section{Author contributions}

E.L. performed the LC-MS analyses, interpreted data, and authored the manuscript. Y.W., E.B., and R.C. assisted with experimental design. E.B. and R.C. developed the SafeMedWaste formulation and R.C. formulated SafeMedWaste for use in experiments. J.S. and M.H. originally conceptualized SafeMedWaste and provided funding and research support.

\section{Competing interests}

The work describes a patented formulation, held by Okra Medical (US patent ID 10668312). JS, YW, RC, EB, and EL are co-inventors on the patent. MH and JS are CEO and CTO of Okra Medical, respectively. Avomeen (employer of EL, YW, RC, and EB) received funding from Okra Medical to perform the research.

\section{Additional information}

Supplementary Information The online version contains supplementary material available at https://doi. org/10.1038/s41598-020-80388-w. 
Correspondence and requests for materials should be addressed to E.L.

Reprints and permissions information is available at www.nature.com/reprints.

Publisher's note Springer Nature remains neutral with regard to jurisdictional claims in published maps and institutional affiliations.

(c) (i) Open Access This article is licensed under a Creative Commons Attribution 4.0 International cc) License, which permits use, sharing, adaptation, distribution and reproduction in any medium or format, as long as you give appropriate credit to the original author(s) and the source, provide a link to the Creative Commons licence, and indicate if changes were made. The images or other third party material in this article are included in the article's Creative Commons licence, unless indicated otherwise in a credit line to the material. If material is not included in the article's Creative Commons licence and your intended use is not permitted by statutory regulation or exceeds the permitted use, you will need to obtain permission directly from the copyright holder. To view a copy of this licence, visit http://creativecommons.org/licenses/by/4.0/.

(C) The Author(s) 2021 